\title{
ÉTUDE PRÉLIMINAIRES SUR L'UTILISATION DES VARIÉTÉS DE BANANIERS RÉSISTANTES DANS LA LUTTE CONTRE LA CERCOSPORIOSE
}

\author{
par J. BRUN \\ Institut Français de Recherches Fruitières Outre-Mer.
}

\begin{abstract}
Les bananiers, rappelons-le brièvement, peuvent être classés en diploïdes à graines et en clones à fruits parthénocarpiques. Ces derniers ont été classés par E. E. CHEESMAN et N. W. SIMMONDS par comparaison avec 1'une des deux espèces ou les deux espèces séminifères qui en sont à l'origine : Musa acuminata (génome A) et Musa balbisiana (génome B). On connaît des variétés diploïdes ou triploïdes qui peuvent être AA, AAA,
\end{abstract} $\mathrm{AAB}, \mathrm{ABB}$.

En ce qui concerne la résistance des bananiers à la Cercosporiose, la plupart des auteurs signalent de grandes variations selon les variétés.

En 1926, CAMPBELL indique qu'au Queensland où sévit une attaque grave, $95 \%$ des bananiers cultivés sont des Cavendish (AAA). SIMMONDS en 1933, rapporte que Gros Michel, Mons Mari, Cavendish (AAA) sont très atteints. Red Dacca (AAA groupe Red) serait résistant, tandis que Lady finger (groupe Pome) et Sugar (groupe Silk), tous deux AAB, montrent une sensibilité variable. En 1935 , WARDLAW citant CAMPBELL indique qu'aux Fidji toutes les variétés commerciales sont atteintes, le champignon aurait été observé sur Sawaga (Musa Fehi).

CHEESMANN et WARDLAW ont étudié la sensibilité des diverses variétés de la collection de l'Impérial Collège à Trinidad. Ils observent que toutes les variétés cultivées sont sensibles à la maladie, mais que la plupart des variétés " sauvages " (Musa acuminata) et leurs hybrides sont résistants.

KERVEGANT signale qu'à Trinidad, les variétés Bluggoe (AAB), Awak legor (ABB), Rajah (ABB),
Palembang (AA) ainsi que Musa acuminata sont résistantes.

WARDLAW indique que pour les variétés résistantes, seules les vieilles feuilles sont tachées, mais ces variétés sont cultivées dans des conditions de disette physiologique extrême, quelques feuilles vivantes peuvent montrer un développement typique des lésions.

WARD indique qu'à la Jamaïque toutes les variétés commerciales sont atteintes; il cite les mêmes variétés résistantes que KERVEGANT à Trinidad.

Au Brésil, DANTAS établit une classification des variétés sensibles. Pas de taches isolées : immunes; présence de stries sans sporulation : hautement résistantes ; taches isolées avec sporulation : résistantes; taches confluentes : sensibles; action sur le régime : hautement sensibles. Dans les conditions brésiliennes, seule $M$. textilis est immune, aucune variété cultivée au Brésil ne peut être considérée comme hautement résistante. "Branca » et "Bacavao " sont résistantes, nous n'avons pas pu rattacher ces deux types à la classification de SIMMONDS. "Inaja» serait la variété la plus sensible.

WOLF écrit qu'au Venezuela toutes les variétés sont sensibles et qu'il n'existe pas de différences spécifiques ou variétales de sensibilité.

HOLLIS signale qu'au Honduras i1 $a$ isolé d'un plantain (AAB ?) des spores qui lui ont donné des colonies identiques à celles obtenues à partir de spores isolées sur Gros Michel et que le plantain peut donc servir de réservoir de spores.

MAGEE indique que toutes les variétés cultivées en Nouvelle-Guinée sont sensibles, seul $M$. textilis est résistant.
ARANGO relate qu'en Colombie Musa paradisiaca (AAB) et Musa cavendishii Lambert(AAA), sont moins sensibles que Musa sapientum Linn. (AAA) ; Musa textilis, M. Fehi Bert. (Australimusa) sont très résistants.

Enfin OCHSE préconise pour la Floride $M$. paradisiaca, variété Hadja (AAB) résistante à la fois à la maladie de Panama et à la Cercosporiose.

\section{SPÉGIFIGITÉ DE MYCOSPHAE- RELLA MUSIGOLA LEACH PAR RAPPORT AUX BANANIERS}

La notion de spécificité en ce qui concerne les Cercospora donne lieu à deux opinions diamétralement opposées. WELLES démontre l'extrême variabilité des conidiophores de différentes espèces de Cercospora étudiées aux Philippines et montre qu'une même espèce, notamment Cercospora lussoniensis Sacc. peut infecter des hôtes différents ; JOHNSON et WALLEAU démontrent également la possibilité d'infections expérimentales de plusieurs espèces à partir d'un même Cercospora. Ils écrivent : "Quand on trouve un Cercospora sur une plante où il n'a pas encore été cité, on croit cela suffisant pour créer une nouvelle espèce. Si un Cercospora a déjà été décrit sur cet hôte, mais que les caractères morphologiques ne correspondent pas, ceci semble justifier la description d'une espèce nouvelle, sans se soucier du fait qu'il peut $y$ avoir des espèces morphologiquement semblables sur beaucoup de plantes appartenant à la même famille ou à des familles différentes ". CHUPP, au contraire, 


\section{Lu dans Fruits, il y a 50 ans ...}

pense que les Cercospora sont remarquablement limités à leur plante-hôte ; quelques-uns, tels ceux décrits sur Solanum, existent sur une espèce d'hôte seulement. S'il était nécessaire de réaliser des inoculations croisées, CHUPP estime qu'elles ne devraient pas être réalisées dans des conditions d'atmosphère saturée au Laboratoire, qui, d'après lui, ne peuvent être acceptées. Les pénétrations des conidies dans de telles conditions sont celles d'un semi-saprophyte.

Nous avons vu d'autre part qu'il existe de très grandes variations dans les dimensions des conidies de Cercospora musae, mais les essais d'inoculation que nous avons établis sur des genres autres que Musa ne nous ont jamais donné de résultats positifs; nous y reviendrons plus loin. CALPOUZOS est arrivé à des résultats identiques, il semble que Cercospora musae $\mathrm{Zimm}$, soit inféodé strictement au genre Musa.

D'autre part, ce même auteur a réalisé des séries de cultures à partir de différents Cercospora récoltés sur des plantes diverses : aucune ressemblance " ni par la morphologie grossière ni par la vitesse de croissance " n'a été trouvée.

A l'intérieur du genre Musa, il existe des variétés immunes et des variétés très sensibles. Le problème de l'influence de la variété sur la germination, la pénétration, n'a jamais été étudié. Nous avons cherché à préciser ces points.

Un premier essai a été mis en place de façon à vérifier la sensibilité de divers bananiers.

Musa acuminata (A).

Figue sucrée (AA).

Bananier nain (AAA).

Banane pomme (AAB).

Les observations effectuées en décembre 1960, 6 mois après leur plantation, nous ont permis d'effectuer sur la neuvième feuille les comptages suivants :

$M$. acuminata : aucune tache.

Figue sucrée : 380 taches par feuille (en moyenne).

Bananier nain: I03 taches par feuille.

Banane pomme : I5 taches par feuille.
Ces observations confirment celles réalisées par d'autres auteurs sur ces variétés.

Nous avons cherché à savoir si cette différence de sensibilité était liée à des phénomènes mécaniques entravant la pénétration, nombre de stomates au centimètre carré par exemple, ou bien s'il existait des différences dans la pénétration et la germination.

I $^{\circ}$ Nombre de stomates au centimètre carré (voir tableau I).

TABLEAU I

Nombre de stomates au centimètre carré (côté gauche, milieu de la feuille).

\begin{tabular}{|c|c|c|}
\hline Clones & Face supérieure & Face inférieure \\
\hline 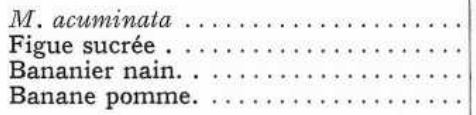 & $\begin{array}{ll}4 & 555 \\
5 & 033 \\
3 & 950 \\
4 & 380\end{array}$ & $\begin{array}{l}\text { I } 8933 \\
\text { I9 } 86 \text { I } \\
\text { I } 4960 \\
\text { I6 } 885\end{array}$ \\
\hline
\end{tabular}

\section{TABLEAU II}

Longueur totale des filaments germinatifs au bout de $24 \mathrm{~h}$.

\begin{tabular}{|c|c|c|c|}
\hline Clones & $\begin{array}{l}\text { Moye } \\
\text { sur les }\end{array}$ & $\begin{array}{l}\text { ueurs } \\
\text { gauche }\end{array}$ & $\stackrel{\%}{\text { de germination }}$ \\
\hline $\begin{array}{l}\text { M. acuminata } \ldots \ldots \ldots \ldots \\
\text { Figue sucrée............... } \\
\text { Bananier nain........ } \\
\text { Banane pomme. } \ldots \ldots \ldots\end{array}$ & $\begin{array}{r}\text { IIO } \\
98 \\
100\end{array}$ & $\begin{array}{r}100 \\
95 \\
83 \\
85\end{array}$ & $\begin{array}{l}95 \\
98 \\
98 \\
97\end{array}$ \\
\hline
\end{tabular}

\section{TABLEAU III}

Pénétration du filament germinatif des ascospores après I20 h.

\begin{tabular}{|c|c|c|c|}
\hline Clones & $\begin{array}{c}\text { Pénétré } \\
\%\end{array}$ & $\begin{array}{c}\text { Non pénétré } \\
\%\end{array}$ & $\begin{array}{c}\text { Non germé } \\
\%\end{array}$ \\
\hline 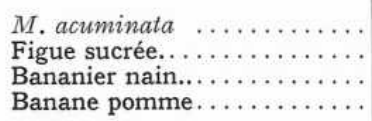 & $\begin{array}{l}66 \\
47 \\
59 \\
54\end{array}$ & $\begin{array}{l}34 \\
53 \\
4 \mathrm{I} \\
46\end{array}$ & $\begin{array}{l}0 \\
0 \\
0 \\
0\end{array}$ \\
\hline
\end{tabular}




\section{Lu dans Fruits, il y a 50 ans ...}

$$
3^{\circ} \text { Pénétration. }
$$

Le même type d'essai a été effectué en étudiant cette fois non plus la germination, mais la pénétration du filament germinatif (tableau III).

On ne relève là non plus aucun rapport entre la pénétration et la sensibilité variétale.

A la suite de ces essais, on peut affirmer que la résistance des différentes variétés de Musa à la Cercosporiose est bien un facteur lié aux conditions du développement du parasite à l'intérieur de la plante.

Il se produit un "blocage » du développement dont le mécanisme nous est totalement inconnu. Le terme de " blocage " nous parait approprié puisque nous savons que, sur des variétés résistantes, des taches peuvent apparaître sur les feuilles sénescentes. Différents auteurs ont signalé, et nous l'avons observé nous-mêmes, 1'apparition de taches sur les vieilles feuilles encore vertes de Musa acuminata considérée comme une variété immune. Il semble que le vieillissement de la feuille permette un développement du parasite sur les variétés résistantes comme l'avait déjà observé WARDLAW.

\section{SENSIBILITÉ A LA GERCOS- PORIOSE DE PLANTES DE} GENRES VOISINS DES MUSA

Nous avons également cherché à observer si des ascospores de $\mathrm{Myco-}$ sphaerella musicola Leach pouvaient germer et pénétrer sur des genres voisins ou sur des plantes d'autres familles. Nous avons retenu les genres suivants :

Ravenala : Strelitziacées (d'après 1a classification de SIMMONDS).

Canna: Cannacées (d'après la classification de SIMMONDS).

Caladium : Aracées.

Les résultats obtenus pour la germination sont donnés dans le tableau IV.

Il est curieux de constater que sur Caladium la germination est très faible, tant par le développement mycélien que par le pourcentage de spores germées après $24 \mathrm{~h}$, alors que sur lames de verre la germination s'effectue normalement, tout au moins au

TABLEAU IV.

Germination des ascospores sur plantes de genre voisin de $M$ usa.

\begin{tabular}{|c|c|c|}
\hline Genres & $\begin{array}{l}\text { Longueur en } \mu \text {. } \\
\text { après } 24 \mathrm{~h} \\
\text { (2 répétitions) }\end{array}$ & \% spores germées \\
\hline 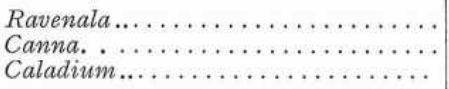 & $\begin{array}{rr}91 & 83 \\
84 & 88 \\
22 & 7\end{array}$ & $\begin{array}{l}96 \\
98 \\
42\end{array}$ \\
\hline
\end{tabular}

TABLEAU $V$.

Essai pénétration du filament germinatif sur plantes de genre voisin de Musa (après I $44 \mathrm{~h}$ ).

\begin{tabular}{|c|c|c|c|c|c|c|}
\hline Genre & \multicolumn{2}{|c|}{$\begin{array}{c}\text { Pénétré } \\
\%\end{array}$} & \multicolumn{2}{|c|}{$\begin{array}{c}\text { Non pénétré } \\
\%\end{array}$} & \multicolumn{2}{|c|}{$\begin{array}{c}\text { Non germé } \\
\%\end{array}$} \\
\hline 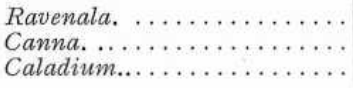 & $\begin{array}{r}60 \\
65 \\
1\end{array}$ & $\begin{array}{r}80 \\
39 \\
0\end{array}$ & $\begin{array}{l}39,5 \\
34 \\
66\end{array}$ & $\begin{array}{l}18 \\
60 \\
54\end{array}$ & $\begin{array}{l}0,5 \\
1 \\
33\end{array}$ & $\begin{array}{r}2 \\
1 \\
46\end{array}$ \\
\hline
\end{tabular}

début (après $24 \mathrm{~h}$, la longueur du filament germinatif est plus élevée que sur feuille en hygrométrie convenable). Peut-être existe-t-il chez le Caladium des substances qui inhibent le développement des ascospores de Mycosphaerella musicola Leach.

Nous avons également cherché à obtenir des pénétrations sur ces trois espèces. Deux essais ont été réalisés sur chaque plante. Les résultats en sont donnés dans le tableau V.

Sur les genres voisins de Musa : Ravenala et Canna, la germination et la pénétration sont normales, mais les symptômes, stries ou taches, n'apparaissent jamais sur les feuilles de ces espèces. Sur Caladium il y a une germination très faible et pas de pénétration.

\section{OBTENTION DES VARIÉTÉS RÉSISTANTES}

Les chercheurs de l'Imperial College à Trinidad essaient depuis de nombreuses années d'obtenir par hybridation des espèces présentant à la fois une résistance à la Cercosporiose et des qualités commerciales correctes. Le but principal de ces travaux est d'obtenir des variétés commerciales résistantes à la maladie de Panama, la résistance à la Cercosporiose venant au second rang.

Ces travaux portent sur trois séries principales d'hybridation.

\section{Hybridation Musa acuminata (mâle) $\times$ Gros Michel (femelle).}

Les hybrides obtenus sont des tétraploïdes du type AAAA. Deux types intéressants ont été retenus :

IC 2 à Trinidad

$\mathrm{S}$ Ig à la Jamaïque.

Le défaut principal de ces hybrides est de présenter des fruits trop courts ; les régimes sont asymétriques et on observe parfois quelques graines. $\mathrm{IC}_{2}$ a néanmoins été utilisé au Honduras comme bananier de village. Ces deux hybrides résistent à la fois à la maladie de Panama et à la Cercosporiose.

Hybridation de AA parthénocarpique (mâle) $\times$ Gros Michel (femelle).

Le parent mâle utilisé est généralement Pisang Lilin. 


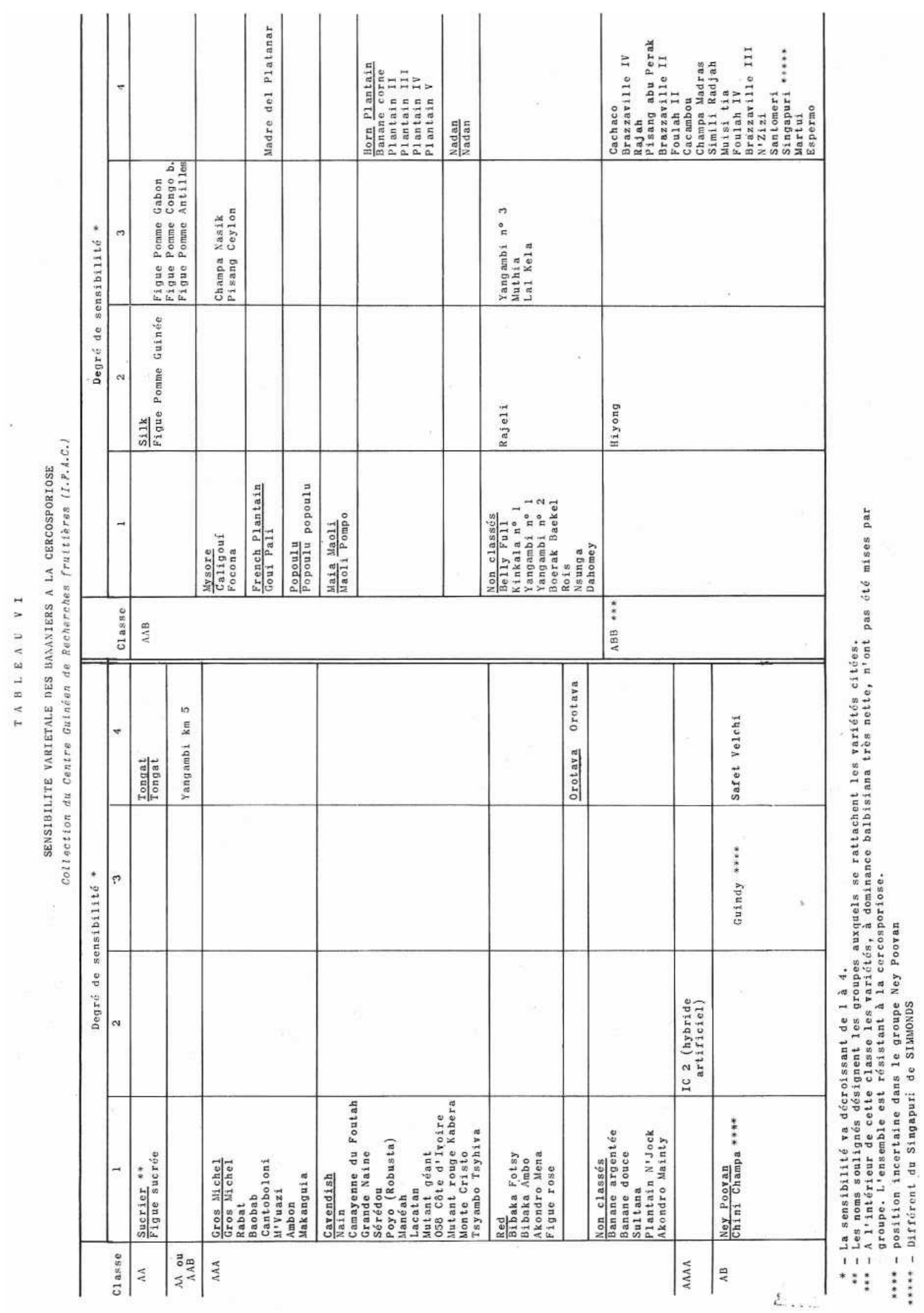




\section{Lu dans Fruits, il y a 50 ans ...}

Les fruits sont beaucoup plus intéressants, proches de ceux de Gros Michel. Le type 1877 donne des rendements équivalents au Lacatan notamment en culture non irriguée ; avec irrigation, ses rendements sont inférieurs à ceux du Lacatan.

La résistance au Cercospora est assez bonne, on admet que les " 1877 " non traités ne sont pas plus atteints que des Lacatan traités à la bouillie bordelaise.

Hybridation entre des parents mâles obtenus par croisement et Gros Michel.

Calcuta $\times$ Pisang Lilin $=$ T I

Calcuta $\times$ Samoa $=\mathrm{SH}_{3}$

Long Tavoy $\times$ Pisang Lilin $=$ 426 A 5

$\mathrm{T} \mathrm{I} \times \mathrm{SH}_{\mathrm{I} 3}=\mathrm{T} \mathrm{I}_{4}-\mathrm{T} \mathrm{I}_{6}-32$

T I $6 \times$ Gros Michel donne des résultats prometteurs, cet hybride est actuellement testé.

$\mathrm{SH} 13 \times 426 \mathrm{~A} 5=\mathrm{T}_{23}$

T $23 \times$ Gros Michel donne également des types très prometteurs actuellement à l'essai.

Il n'est pas possible d'envisager des hybridations à partir des variétés du type Cavendish qui intéresseraient le marché français, car la production de graines s'est avérée nulle dans tous les essais entrepris.

Utilisation de variétés résistantes comme bananiers de village.

Une des difficultés rencontrées dans 1a lutte systématique contre la Cercosporiose est la présence dans les villages de touffes de bananiers atteints par la maladie, qui sont impossibles à traiter à cause de leur dispersion et que les villageois ne veulent à aucun prix détruire. Nous avions demandé aux agronomes du Centre guinéen de Recherches fruitières la multiplication d'une variété appelée Yangambi $\mathrm{km} 5$ ( $\mathrm{AA}$ ou $\mathrm{AAB}$ ?) qui résiste à la Cercosporiose et qui fournit des fruits de dessert corrects. Nous n'avons absolument pas réussi à implanter cette variété. En effet, les fruits des bananiers de case appartiennent aux femmes qui, en les vendant au marché, obtiennent un gain personnel. Les femmes se sont toujours refusées à la substitution de la Yangambi aux variétés sensibles : Canaries, Poyo ou Manéah (toutes des AAA) habituellement utilisées.

\section{SENSIBILITÉ VARIÉTALE A LA GERGOSPORIOSE}

SIMMONDS a établi un tableau général de la sensibilité à la Cercosporiose des variétés de bananiers. Il utilise la cotation suivante : $\mathrm{I}$, sensible,

à 4 , très résistant. Nous avons effectué un travail similaire à partir d'observations effectuées sur la collection du Centre guinéen de Recherches fruitières (tableau VI).

Nous nous sommes efforcés de classer les variétés selon la méthode de N. W. SIMMONDS. Cette opération est relativement aisée pour les groupes à caractère acuminata : diploïdes AA, triploïdes AAA. En ce qui concerne les $\mathrm{AAB}$ et les $\mathrm{ABB}$, nous admettrons avec J. CHAMPION (généticien à

TABLEAU VII

Sensibilité variétale des bananiers à la Cercosporiose. Collection de la Station I. F. A. C. de Neufchâteau en Guadeloupe, Antilles.

\begin{tabular}{|c|c|c|c|c|}
\hline \multirow[t]{2}{*}{ Classe } & \multicolumn{4}{|c|}{ Sensibilité à la Cercosporiose } \\
\hline & I & 2 & 3 & 4 \\
\hline $\mathrm{AA}$ & $\begin{array}{l}\text { Sucrier : } \\
\text { Figue sucrée } \\
\text { Fraysinette }\end{array}$ & & & $X$ : \\
\hline AAA & $\begin{array}{l}\text { Gros Michel: } \\
\text { Highgate } \\
\text { Weeping Jamaï- } \\
\text { ca } \\
\text { Raimbaud } \\
\text { Plantain Jamaï- } \\
\text { ca } \\
\text { Cavendish: } \\
\text { China } \\
\text { Mardi-Gras } \\
\text { Red: } \\
\text { Figue rose }\end{array}$ & $\begin{array}{l}\text { Red : } \\
\quad \text { Tisane (2-3) } \\
\quad \text { Tisane blanche }\end{array}$ & & \\
\hline $\mathrm{AAB}$ & $\begin{array}{l}\text { French Plantain : } \\
\text { Banane roo li- } \\
\text { vres } \\
\text { Banane puce } \\
\text { Ces deux variétés } \\
\text { sont intermé- } \\
\text { diaires entre I } \\
\text { et } 2\end{array}$ & $\begin{array}{l}\text { Silk: } \\
\text { Figue pomme } \\
\quad(2-3) \\
\text { Divers : } \\
\text { Mysore (I-2) } \\
\text { Bananes } 7 \text { se- } \\
\text { maines } \\
\text { Nendra padath- } \\
\text { thi (I-2) }\end{array}$ & & \\
\hline $\mathrm{ABB}$ & & & & $\begin{array}{l}\text { Bluggoe : } \\
\text { Cacambou } \\
\text { Poteau rose }(3-4) \\
\text { Poteau blanche }\end{array}$ \\
\hline
\end{tabular}

NB : Les noms en italiques désignent les groupes auxquels se rattachent les variétés citées. La sensibilité va décroissant de I à 4 . 


\section{Lu dans Fruits, il y a 50 ans ...}

TABLEAU VIII

Réaction de différents clones à la Cercosporiose.

(Tableau extrait de 1'ouvrage de SIMMONDS, Bananas, p. $3^{85}$.)

\begin{tabular}{|c|c|c|c|c|}
\hline \multirow[t]{2}{*}{ Classe } & \multicolumn{4}{|c|}{ Sensibilité à la Cercosporiose } \\
\hline & I & 2 & 3 & 4 \\
\hline AA & $\begin{array}{l}\text { Sucrier } \\
\text { Bande } \\
\text { Sikuzani }\end{array}$ & & Mchare & $\begin{array}{l}\text { Tongat } \\
\text { Pisang lilin } \\
\text { Palembang } \\
\text { Paka }\end{array}$ \\
\hline AAA & $\begin{array}{l}\text { Groupe Cavendish } \\
\text { Gros Michel } \\
\text { Marathuva (2 ?) } \\
\text { Palimbang }\end{array}$ & Rio & $\begin{array}{l}\text { Red } \\
\text { Green Red } \\
\text { Groupe Luju- } \\
\quad \text { gira }\end{array}$ & Orotava \\
\hline AAAA & & & & IC 2 \\
\hline $\mathrm{AB}$ & & & & Ney poovan \\
\hline $\mathrm{AAB}$ & & $\begin{array}{l}\text { Nendra padaththi } \\
\text { Rajapuri }\end{array}$ & $\begin{array}{l}\text { Pisang Rajah } \\
\text { (4 ?) } \\
\text { Pome } \\
\text { Nadan } \\
\text { Silk }\end{array}$ & $\begin{array}{l}\text { Mysore } \\
\text { Pisang Kelat } \\
\text { Groupe Plan- } \\
\quad \text { tain }\end{array}$ \\
\hline ABB & & & & $\begin{array}{l}\text { Bluggoe } \\
\text { Peyan } \\
\text { Pacha bontha } \\
\text { bathees } \\
\text { Pisang awak } \\
\text { Ney mannan }\end{array}$ \\
\hline ABBB & & & & Klue teparod \\
\hline
\end{tabular}

1'I. F. A. C.) qu'il s'agit de groupes respectivement à dominance acuminata et à dominance balbisiana. Il est intéressant de rapporter ici le travail que nous avons établi en collaboration avec P. FROSSARD, car il existe, pour les variétés dont la position est bien établie, des différences assez grandes avec la sensibilité que leur confère SIMMONDS.
Nous avons également fait des observations similaires sur la collection de la Station I. F. A. C. de Neufchâteau aux Antilles (tableau VII).

Enfin, nous donnons le tableau de SIMMONDS (tableau VIII) afin de permettre la comparaison avec nos observations effectuées en Guinée ; par groupe nos observations concordent avec celles de SIMMONDS.
Alors que SIMMONDS classe les Red et les Green red en 3, nous les avons notés $I$; il existe peut-être une faible différence mais, sous les conditions guinéennes, la sensibilité des Red est beaucoup plus importante que ne l'indique cet auteur.

AAAA. IC-2 est également moins résistant en Guinée qu'à Trinidad ou à la Jamaïque.

AB. Chini Champa (cette espèce a été introduite en collection à partir des Indes, elle semble bien être une $\mathrm{AB}$ ) est très sensible alors que SIMMONDS considère que les "Ney poovan " en général sont résistants.

$\mathrm{AAB}$. Ce groupe présente une très grande hétérogénéité quant à sa résistance à la Cercosporiose ; cependant, à une exception près, Nadan, que nous plaçons en 4 alors que SIMMONDS lui attribue la note 3 , toutes les variétés ont, ou bien des notes identiques, ou bien des notes inférieures : un assez grand nombre de clones sont classés I dans nos observations, alors que les plus atteints sont classés 2 chez SIMMONDS ; enfin, certaines variétés rangées parmi les Silk ont été cotées I par nous alors que SIMMONDS range les Silk en catégorie 3 .

ABB. Dans 1'ensemble, ce groupe est résistant aussi bien en Guinée que d'après SIMMONDS; une seule exception : Hiyong. Ce bananier originaire de la République gabonaise nous semble bien un $A B B$, il est possible que nous ayons commis une erreur dans la position de cette variété.

Dans l'ensemble, on peut admettre que, pour la plupart des variétés considérées comme moyennement résistantes, la sensibilité à la Cercosporiose est augmentée dans les conditions guinéennes.

Centre guinéen de Recherches fruitières.

Extrait du Rapport Annuel 1960-6I de l'Institut Français de Recherches Fruitières Outre-Mer (I. F. A. C.) 
BIBLIOGRAPHIE

Arango, B. H. - La Sigatoka del banano, Cercospora musae Zimm. Acta agron. Colombie, oct. $1956,6,4$, p. $159-172$.

Calpouzos, L. - Studies on the Sigatoka disease of bananas and its fungus pathogen; Atkins Garden and Research Laboratory, Cienfuegos (Cuba), I955, $70 \mathrm{p}$.

CAmpbell, J. G. C. - Banana disease (Report by the Government Mycologist on a visit to Queensland to investigate). Legislature council, Fiji, 1926, Council Paper $n^{\circ} 32$.

Chensman, E. E. et Wardeaw, C. W. - Specific and varietal susceptibility of bananas to Cercospora leaf-spot. Trop. A gric. Trinidad, 1937, I4, I2, p. 335-336

CHupp, C. - A monograph of the fungus genus Cercospora; Ithaca (New York), 1953, $667 \mathrm{p}$.

Dantas, B. - A ocorrencia da "Cercosporiose "da bananeira no Brasil, Cercospora musae Zimm. Bol. tec. Inst. agron. Norte, mars $1948, \mathrm{r} 4,46 \mathrm{p}$.

Hollis, J. P. - Sigatoka disease of banana; non publié. Dept. Trop. Res. United Fruit Co, La Lima, Honduras, mai 1953 , mars I954.

Johnson, E. M. et WAlleaU, W. D. - Synonymy in some common species of Cercospora. Phytopath., 1953, 43, p. 477.

Kervegant, D. - La maladie à Cercospori: des feuilles du bananier. Imprimerie du Gouvernement, Fort-de-France, 1938, I6 p.
Magee, C. J. - Outreaks and new records. F. A. O. Pl. Prot, Bull., 1954, 2, 10, p. 154-157.

Ochse, J. J. - A new banana for Florida, Musa paradisiaca L. var. Hadja. Proc. Fla. St. Hort. Soc., 1957, 7o, p. 340-34r. Simmonds, J. H. - Banana leaf-spot. Queensland Agric. J., Janv. I933, 39, p. 2I-40.

Simmonds, N. W. - Bananas. Lonton, Longmans, r959, I $^{\text {re }}$ éd., $466 \mathrm{p}$.

WARD, F. S. - Factors affecting the control of Cercospora musae. J. Jamaïca Agric. Soc., Oct. I939; Dept. Sci Agric. Jamaïca, Reprints of Misc. Articles I939, Bull. no 27 , 4 p.

Wardlaw, C. W. - Diseases of the banana, Lcndon, Mac Millan, $\mathrm{r} 935, \mathrm{I}^{\mathrm{re}}$ ed., $6 \mathrm{I} 5 \mathrm{p}$.

Wardlaw, C. W. - Cercospora leaf-spot. Disease of bananas. Nature, Jul. 1939, I44, p. Ir.

Welles, C. B. - Observations on taxonomic factors used in the genus Cercospora. Science, Janv. Jun. 1924, new series, 59, p. $216-218$.

Welles, C. B. - Taxonomic studies on the genus Cercospora in Philippines Islands. Amer. J. Bot., 1925, I2, p. I95-218.

Wolr, F. A. - Notes on Venezuelan fungi. Lloydia, 1949, 12, 4, p. 208-2r9. 